Disponível em

ANRAD|

http://www.anpad.org.br/rac

RAC, Rio de Janeiro, v. 17, n. 2, art. 3, pp. 176-197, Mar./Abr. 2013

(cc) EY-NC

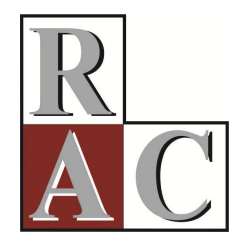

\title{
Casos de Ensino no Brasil: Análise Bibliométrica e Orientações para Autores
}

Teaching Cases in Brazil: a Bibliometric Analysis and Guidelines for Authors

Marina Faria

E-mail: marinafaria86@hotmail.com Universidade Federal do Rio de Janeiro - COPPEAD/UFRJ Rua Pascoal Lemme, 355, Ilha do Fundão, 21941-918, Rio de Janeiro, RJ, Brasil.

Klebler Fossati Figueiredo

E-mail: kleber@coppead.ufrj.br

Universidade Federal do Rio de Janeiro - COPPEAD/UFRJ Rua Pascoal Lemme, 355, Ilha do Fundão, 21941-918, Rio de Janeiro, RJ, Brasil. 


\title{
Resumo
}

A demanda pela utilização de métodos participativos de ensino vem aumentando em cursos de administração. Correspondentemente, cresce o número de professores que utilizam esse método por meio de casos. Como forma de incentivar a disseminação de casos brasileiros, em 2007, a Associação Nacional de Pós-Graduação e Pesquisa em Administração (ANPAD) passou a aceitar casos de ensino em seus congressos. A presente pesquisa tem por objetivo propor diretrizes para acadêmicos brasileiros em administração que desejem produzir casos de ensino, a partir de um panorama da produção dos casos nacionais publicados entre 2007 e 2011 . Foi realizada uma análise bibliométrica acerca das publicações nos eventos da ANPAD e nos principais periódicos nacionais. Os resultados apontaram que os maiores problemas ocorrem nas notas de ensino. A maioria dos casos é considerada pelos autores como útil para as áreas de estratégia e de marketing, porém há áreas que contam com poucos casos, como gestão de pessoas, logística e finanças. A análise alertou para a predominância de um perfil de protagonista e para a falta de diversificação das fontes de dados utilizadas para a elaboração dos casos. O artigo traz 16 diretrizes com o propósito de orientar acadêmicos brasileiros que desejem produzir casos de ensino em administração.

Palavras-chave: casos de ensino; bibliometria; diretrizes.

\begin{abstract}
The demand for the use of active teaching methods is increasing in management courses. Correspondently, a growing number of teachers are using the case method. In order to encourage the spread of cases in Brazil, the Associação Nacional de Pós-Graduação e Pesquisa em Administração (ANPAD - Brazilian Academy of Management) began accepting teaching cases in its conferences in 2007. This research aims to propose guidelines for Brazilian scholars who wish to produce teaching cases based upon a panorama of national cases published between 2007 and 2011. We performed a bibliometric analysis of publications in ANPAD sponsored events and major national journals. The most common problems related to teaching notes. The majority of cases are indicated by the authors as useful in the areas of strategy and marketing, but there are areas with few cases, such as people management, logistics and finance. Our analysis drew attention to the predominance of a player profile and lack of diversification in data sources used for case preparation. This article provides 16 guidelines for Brazilian scholars who wish to producing teaching cases in management.
\end{abstract}

Key words: management teaching cases; bibliometrics; guidelines. 


\section{Introdução}

Os métodos tradicionais de ensino, embasados principalmente em aulas expositivas, não raramente são criticados por negligenciar a formação pragmática e o desenvolvimento do espírito experimental entre os alunos (Mumford, 2005), principalmente quando o processo educacional envolve indivíduos adultos (Rosier, 2002). A esse respeito, as perspectivas didáticas fundamentadas no pragmatismo e no construtivismo apregoam ser fundamental o papel ativo do estudante no processo de aprendizagem (Machado \& Callado, 2007; Vergara, 2003). Nos métodos ativos de ensino, que se opõem aos ortodoxos, a participação dos alunos é crucial, e deve ser incentivada pelos professores que exercem os papéis de mediadores da aprendizagem (Garvin, 1991; Oros, 2007). Para Apaydin (2008), os métodos educacionais participativos estimulam simultaneamente as modalidades comportamental e cognitiva da aprendizagem, conduzindo o estudante a níveis mais elevados de fruição do conhecimento e de aquisição de habilidades.

Em cursos de graduação e de pós-graduação em administração, os meios pedagógicos ativos ganharam espaço principalmente em resposta à preocupação cada vez maior de desenvolver nos alunos a habilidade de lidar com a complexidade dos ambientes nos quais as decisões empresariais são tomadas (Ambrosini, Bowman, \& Collier, 2010; Banning, 2003). Um método ativo muito utilizado por professores de administração é o método do caso, que foge do padrão de ensino centrado no docente, e coloca o aluno como principal agente de seu aprendizado (Duarte, 2010; Garvin, 2007; Greenhalgh, 2007). Esse método baseia-se na ideia de que aprender e ensinar fazem parte de um mesmo contínuo (Christensen, 1991; Levin, 1995). Almeida e Figueiredo (2007), por instância, afirmam que, durante o estágio de preparação individual do caso, o aluno inicia um importante processo de descobrimento. Para Machado e Callado (2007), o aprendizado pela descoberta se origina do esforço analítico do estudante para separar dados úteis e inúteis, interpretar e encontrar significados nos fatos que compõem o caso.

No Brasil, Kruel e Santos (2008) apontam que o interesse pelo método do caso parece ter ganho novo fôlego devido à proliferação de cursos de pós-graduação lato sensu e de mestrados profissionais em administração. Não obstante tais demandas, e a despeito das crescentes evidências sobre a importância da utilização do método do caso em escolas de administração, Ikeda, Veludo-de-Oliveira e Campomar (2005) e Roesch (2007) apontam que a produção nacional de casos para ensino ainda é muito pequena. Assim, buscando suprir a demanda por casos que relatem situações vivenciadas por executivos no contexto brasileiro, desde 2007 a Associação Nacional de Pós-Graduação e Pesquisa em Administração (ANPAD) publica casos de ensino nos anais de seu congresso mais expressivo, o Encontro Nacional da Associação Nacional de Pós-Graduação e Pesquisa em Administração (EnANPAD).

Tomando esse cenário em perspectiva, a presente pesquisa tem por objetivo propor diretrizes para acadêmicos brasileiros em administração que desejem produzir casos de ensino a partir de um panorama da produção dos casos brasileiros publicados entre 2007 e 2011. Para cumprir com tal objetivo, foi realizada uma análise de base bibliométrica acerca das publicações nos eventos promovidos pela ANPAD e nos principais periódicos nacionais especializados em administração entre os anos de 2007 e 2011. A abordagem bibliométrica forneceu, portanto, o suporte metodológico para que fosse possível descrever e analisar as características dos casos de ensino publicados. No que se refere à delimitação da pesquisa, cabe observar que Böcker (1987) classifica os casos de ensino em dois grandes grupos, sendo que o presente artigo concentra seu foco nos casos correspondentes ao primeiro grupo: (a) casos-problema, associados ao método do caso, concebido na Universidade de Harvard; e (b) casos-demonstração, utilizados para ilustrar aulas expositivas, e que aqui não são abordados.

Este artigo foi estruturado em sete seções, das quais esta introdução é a primeira. Na segunda seção, apresenta-se a revisão de literatura que fundamentou a análise dos casos de ensino coletados nos periódicos e nos anais dos eventos da ANPAD. A terceira seção apresenta um quadro que resume 
os critérios apontados pela literatura que serviram de base para a análise dos casos. A quarta delineia os aspectos metodológicos da pesquisa. Na quinta, mostram-se a caracterização e a análise dos casos, para que, na sexta, sejam sugeridas 16 diretrizes a serem eventualmente consideradas por acadêmicos brasileiros em administração que tenham interesse em produzir casos de ensino. Na sétima seção do artigo, são apresentadas as considerações finais e as sugestões para futuras pesquisas sobre o tema.

\section{Revisão de Literatura}

\section{Casos de ensino: aplicações, vantagens e dificuldades}

Como recordam Mahboubian (2010) e Roesch (2007), o método do caso vem sendo utilizado na formação gerencial há cerca de um século em universidades norte-americanas e, mais recentemente, na Europa e na Ásia. Ferlie, McGivern e Moraes (2010, p. 60) argumentam que o método do caso desenvolvido em Harvard foi "um legado passado a partir das escolas de direito para as escolas de administração". No Brasil, os casos de ensino ainda são pouco aproveitados, e é pequeno o volume de publicações de casos brasileiros, mas vem crescendo sobremaneira a demanda por esse recurso pedagógico. Roesch (2007) alerta que a bibliografia em português sobre o assunto é escassa, e que muitos professores confundem casos para ensino com estudos de caso, o que faz com que os casos brasileiros muitas vezes não tenham os elementos necessários para suscitar o aprendizado.

Um caso para ensino é a descrição de uma situação real com objetivos educacionais, comumente envolvendo uma decisão, um problema ou uma oportunidade vivida por alguém em uma organização (Mauffette-Leenders, Erskine, \& Leenders, 2007; Roesch \& Fernandes, 2007). Por meio dos casos, o aluno pode vivenciar situações similares às que são encontradas nas empresas, mas sem correr os riscos que estão relacionados às decisões a serem tomadas no mundo real (Suarez \& Casotti, 2004; Wang \& Wang, 2011). Casos de ensino costumam ser amplamente utilizados nos programas de educação para gestão e negócios, pois permitem que os estudantes aprendam por meio da aplicação e da adaptação dos conceitos teóricos às situações específicas descritas (Ambrosini et al., 2010; Christensen \& Carlile, 2009).

Visando esclarecer as diferenças entre casos de ensino e estudos de caso, Roesch e Fernandes (2007) apontam que os estudos de caso têm seu objetivo direcionado para a pesquisa acadêmica, enquanto os casos de ensino objetivam promover a aprendizagem; por conseguinte, o público-alvo dos estudos de caso são os acadêmicos, enquanto o dos casos de ensino são os alunos. Por esse mesmo motivo, casos de ensino devem ser desenvolvidos tendo em vista os interesses e as necessidades dos alunos (Bess, 2004; Leenders, Mauffette-Leenders, \& Erskine, 2001; Sebenius, 2011). Envolver os alunos é fundamental, uma vez que a utilização do método do caso só faz sentido se os alunos participarem (Bontis, Hardie, \& Serenko, 2009; Duarte, 2010; Ellet, 2007; Gil, 2004). Roberts (1999) aponta que um caso precisa ter uma história envolvente para que o aluno de fato participe das discussões, enquanto Roesch e Fernandes (2007) afirmam que o estilo da redação dos casos de ensino deve ser atraente a fim de que os alunos se envolvam com as decisões; para tais autores, imprimir um tom de mistério na narrativa dos casos de ensino pode ser interessante. Roesch (2007) compara os casos de ensino com textos jornalísticos, alertando para que o texto traga fatos e depoimentos apresentados em ordem cronológica, com o propósito de proporcionar uma discussão bem informada do caso em sala de aula.

Um dos motivos pelos quais os alunos podem vir a não se interessar por um caso de ensino está relacionado à percepção de que seus dados e problemas possam eventualmente aparentar ser muito antigos, havendo uma necessidade constante de que casos novos sejam produzidos (Erskine, Leenders, \& Mauffette-Leenders, 2003). No entanto, desenvolver um caso de ensino pode ser complicado, principalmente em razão da dificuldade de fazer com que uma empresa se disponha a fornecer os dados necessários para sua elaboração. As empresas que autorizam que o caso seja redigido, divulgado e debatido podem ter interesse legítimo em colaborar para a educação de futuros gerentes, ou podem 
enxergar nos casos uma oportunidade para recrutar estudantes que se interessem em trabalhar na organização depois de conhecê-la por meio do caso (Corey, 1999). Na prática, os gestores das empresas autorizam apenas os casos que contenham informações que eles julgam ser positivas para a imagem de suas organizações (Roberts, 1999). Devido a tais impedimentos e dificuldades impostos pelas empresas, muitos casos são desenvolvidos somente porque seus autores conhecem funcionários que se prontificam a fornecer as informações, conquanto o ideal fosse que os casos surgissem das necessidades dos professores e estivessem alinhados com objetivos de ensino.

Garvin (1991) aponta que as dificuldades para uma utilização melhor e mais ampla do método do caso passam pela resistência à mudança por parte de todos os envolvidos: existem professores que não se sentem confortáveis com o método e há instituições de ensino que, por discordância em função de questões pedagógicas, não endossam processos de ensino-aprendizagem por meio de casos. Ademais, a resistência também se faz notar por parte dos próprios alunos, que muitas vezes acreditam que irão aprender menos em disciplinas que se valem do método do caso, pois os docentes irão falar menos. No que diz respeito aos professores, Christensen (1991), Levin (1995) e Roberts (1999) acreditam que a prática faz com que os docentes sintam-se mais à vontade para ministrar aulas utilizando o método.

Machado e Callado (2007) alertam que não se pode tomar o método do caso como panaceia para resolver os problemas intrínsecos à aprendizagem, nem tampouco acreditar que seu uso seja suficiente para que os alunos possam produzir conhecimentos que lhes capacitem a entender situações que extrapolem as premissas contidas nos casos. Para esses autores, quando o aluno se defronta com informações dispostas de forma sistematizada e clara, cria-se a falsa impressão de que as mesmas são de fácil obtenção. Iizuka (2008) defende ser preciso refletir sobre as adaptações necessárias para a utilização do método do caso no Brasil, para não incorrer no equívoco de reproduzir de modo acrítico e sem contextualização histórica, política, social e econômica um método norte-americano de ensino.

\section{Desenvolvimento e redação de casos para ensino}

Para Ikeda et al. (2005), é inquestionável a falta de casos locais que abordem problemas de empresas brasileiras ou de empresas estrangeiras no Brasil. Diante dessa carência, é importante que acadêmicos brasileiros preocupem-se em desenvolver e redigir casos de ensino adequados à realidade do país. Nesse sentido, Kruel e Santos (2008) defendem que alunos de pós-graduação em administração deveriam ser instruídos a respeito da forma pela qual um caso de ensino deve ser escrito. Roesch e Fernandes (2007) e Webb, Gill e Poe (2005) recordam que os casos de ensino precisam ter objetivo pedagógico e que o estilo da redação dos casos deve ser atraente para que os alunos se envolvam com as decisões.

Assim, é importante considerar a motivação dos alunos no momento da elaboração de um caso. Um dos fatores que pode tornar o caso mais atraente é a escolha de um protagonista com o qual os alunos tenham identificação. Dois exemplos ilustram essa afirmação.

No caso Sothebys.com (Hallowell \& Hansen, 2000, p. 2), há na introdução uma frase que identifica o protagonista como um ex-aluno de um programa de mestrado: "Estamos criando os melhores sites de arte e antiguidade do mundo. Se você estiver procurando itens sofisticados e autênticos, é conosco mesmo. A qualidade do que vendemos e nossa experiência torna-nos únicos" (Craig Moffet, MBA da HBS em 1989 e presidente da Sothebys.com).

Já o caso Hewlett-Packard: Deskjet Printer Supply Chain (Kopczak \& Lee, 1996, p. 1) começa com os autores associando o protagonista com uma prática esportiva que possivelmente encontra adeptos entre os estudantes: "Brent Cartier, gerente de projetos especiais da Divisão Vancouver da HP, completou outra milha pedalando sua bicicleta. Tinha sido uma longa semana, e parecia que seria um longo final de semana também, porque precisava preparar a reunião que teria na segunda-feira sobre os níveis mundiais de estoques da impressora Deskjet”. 
O estudante precisa estar bastante envolvido com a história apresentada para que de fato participe da discussão (Christensen, 1991; Levin, 1995), haja vista que, para que o método do caso seja efetivo, a participação dos alunos é fundamental (Bontis et al., 2009; Brennan \& Ahmad, 2005; Siciliano \& Mcaleer, 1997). Esse envolvimento só é obtido se o caso retratar problemas do dia a dia dos alunos (Corey, 1998), e se houver identificação com o protagonista do caso, aquele que deve tomar as decisões (Franke, 2011; Sebenius, 2011). Para ilustrar esse ponto, há autores que defendem a posição de que os casos aplicados em turmas de pós-graduação devem ter protagonistas pós-graduados (Roberts, 1999). Leenders, Mauffette-Leenders e Erskine (2001) alertam que a determinação acerca de quem será o tomador de decisão na narrativa de um caso para ensino é uma das escolhas mais importantes a serem tomados pelo autor do caso, uma vez que, se essa escolha for mal sucedida, o caso pode tornar-se extremamente desinteressante para os alunos. Note-se, adicionalmente, que, a despeito de a maioria dos casos centrarem-se na figura de um executivo principal (Swiercz \& Ross, 2003), tanto Roberts (1999) quanto Sebenius (2011) constatam que são muito mais realistas os casos que trazem outros pontos de vista além daquele do protagonista ou decisor principal.

Abell (1997) enumera dez características essenciais para que os casos cumpram sua função como instrumentos de ensino: (a) conter uma ou mais questões gerenciais a serem confrontadas e selecionadas, por meio de debate; (b) tratar de tópicos relevantes para a área; (c) proporcionar descobertas; (d) levantar controvérsias que possam ensejar diferentes interpretações, decisões e planos de ação; (e) conter contrastes e comparações; (f) permitir aos alunos generalizar lições e conceitos subjacentes ao caso para outras situações; (g) conter dados apropriados para tratar dos problemas, tais como descrição dos produtos, indústrias, pessoas envolvidas e dados quantitativos; (h) conter uma narrativa que guarde um toque pessoal, incluindo falas dos participantes e descrições de processos organizacionais formais e informais; (i) ser bem estruturados e bem relatados; (j) ser curtos, contendo no máximo de oito a dez páginas de texto e até cinco páginas de anexos.

Para Leenders et al. (2001), os títulos dos casos para ensino não devem ser engraçados e, preferencialmente, um caso deve ser identificado somente pelo nome da empresa. Roesch (2007) recomenda que os títulos e subtítulos sejam neutros, uma vez que os casos devem conter relatos imparciais. Pinnington (2005), a seu turno, acredita que usar humor em doses parcimoniosas pode favorecer o processo de aprendizagem, enquanto Gil (2004) aconselha a evitar a ironia na redação de casos.

Segundo Roberts (1999) e Roesch (2007), no primeiro parágrafo de um caso - ou ao menos em sua primeira página - devem vir descritos os pontos mais importantes para sua análise: quem é o protagonista, quais as principais questões, em que empresa o caso se passa e quando se dão seus acontecimentos. Swiercz e Ross (2003) apontam o primeiro parágrafo como o mais rico de todo o caso, e Leenders et al. (2001) apresentam um checklist para esse parágrafo. Para os autores, o parágrafo inicial deve conter o nome do protagonista e de seu cargo, além de situar o leitor com respeito à organização em questão e sobre o tempo durante o qual se passa o caso. No parágrafo final, a situação problema apresentada no primeiro parágrafo é retomada, e o aluno é convidado a refletir sobre a situação.

A confidencialidade constitui uma das principais barreiras para se escrever um caso, pois, de modo geral, as empresas temem que seus dados sejam utilizados pelos concorrentes. Uma forma de atenuar essa percepção de risco é mudar o nome da empresa, os dados financeiros disponibilizados na narrativa e até mesmo os nomes dos personagens (Roberts, 1999). É importante alertar que o autor deve obter a autorização da empresa, mesmo que o nome da mesma esteja camuflado (Roesch \& Fernandes, 2007).

Outra dificuldade encontrada pelos autores de casos é a definição do tamanho ideal que deve ter o caso, e a esse respeito registram-se discordâncias entre diversos autores. Para Corey (1998), curiosamente, um caso de cinco páginas é capaz de gerar mais discussão do que um caso de 25 ou 50 páginas. Ikeda et al. (2005) apontam que os alunos tendem a preferir casos curtos e recentes. Abell (1997) também defende que os casos sejam curtos, enquanto Roberts (1999) aponta que normalmente 
os casos têm entre sete e doze páginas. Para esse autor, quanto mais tempo o aluno precisa investir para ler o caso, menos tempo terá para pensar sobre o caso.

No que diz respeito à redação dos casos de ensino, Roberts (1999) e Sebenius (2011) defendem que termos técnicos devem ser evitados; caso seja fundamental incluí-los no texto, então eles devem ser definidos à medida que aparecerem. É preciso ter cuidado também com o tempo verbal no qual o caso é escrito: para que fique menos datado, Gil (2004) e Leenders et al. (2001) propõem que ele seja escrito no tempo passado. Para Roesch (2007), contudo, a dificuldade de redigir um caso para ensino deve-se principalmente ao processo de vaivém entre presente e passado, e à necessidade de se introduzir transições na narrativa para salientar tais momentos. Por fim, outro ponto importante na redação de casos é a imparcialidade do autor: sempre que o caso apresentar falas dos personagens, o autor não deve se posicionar a respeito, permitindo que o leitor imprima seu próprio julgamento (Roesch \& Fernandes, 2007).

\section{A elaboração das notas de ensino}

As notas de ensino constituem uma parte muito importante nos casos (Cappel \& Schwager, 2002; Corey, 1999; Lundberg \& Winn, 2005), pois têm a finalidade de fornecer esclarecimentos ao docente que irá utilizar esse recurso didático: elas "auxiliam na verificação da adequação do caso e na definição de estratégias para o seu desenvolvimento", além de também servirem para prover "informações adicionais sobre o caso, o que contribui para que o professor tenha maior segurança na condução das discussões" (Gil, 2004, p. 15). Roberts (1999) assevera que não existem regras rígidas para a construção das notas de ensino. Roesch e Fernandes (2007) destacam que há divergências até mesmo sobre em que momento devem ser incluídas essas notas. Roesch (2007), contudo, aponta que as notas devem conter ao menos um resumo do caso, as fontes de dados, os objetivos educacionais, as alternativas para a análise do caso, algumas questões para discussão e a bibliografia recomendada para a análise do caso.

Por ser o caso uma situação real, é fundamental que o autor deixe claro quais foram as fontes usadas para construir a narrativa (Gil, 2004; Roesch, 2007). Corey (1999) e Roberts (1999) enfatizam que é importante para o autor do caso buscar fontes públicas de informação, de modo que a narrativa não traga apenas o ponto de vista da empresa. Roberts (1999) novamente acredita também ser crucial que o autor consiga ter acesso aos documentos da empresa. Leenders et al. (2001) focam na importância de aproveitar as entrevistas para entender o máximo possível da situação do caso. Para Ikeda et al. (2005) e Roberts (1999), é importante entrevistar várias pessoas que ocupem diferentes posições na organização. Corey (1999) é a favor de entrevistas não estruturadas, e defende que o mesmo informante deve ser entrevistado ao menos duas vezes.

Lundberg e Winn (2005) e Sebenius (2011) creem que uma abordagem minuciosa das notas de ensino traz valiosos incrementos na forma pela qual um caso é previamente preparado por um professor, bem como nas discussões que se seguirão em classe. Para tanto, é fundamental que as notas tragam os objetivos de ensino do caso (Corey, 1999), uma vez que a descrição de tais objetivos é a parte mais importante das notas de ensino (Austin, 1993): por meio deles, os professores que tencionam valer-se do caso entrarão em contato com os assuntos que podem ser abordados por intermédio daquele caso (Roberts, 1999).

Roesch e Fernandes (2007) dividem os objetivos educacionais em dois domínios: o cognitivo, que envolve conhecimento, compreensão, aplicação, análise, síntese e avaliação; e o domínio afetivo, que está relacionado com emoções, atitudes e apreciações. Para esses autores, os principais objetivos educacionais dos casos são: (a) desenvolver habilidades e atitudes consideradas chaves para o bom desempenho gerencial; (b) familiarizar os estudantes com as organizações e seu ambiente; e (c) ilustrar teorias, modelos ou conceitos.

Roberts (1999) aponta que as notas de ensino devem conter perguntas para orientar os docentes, as quais podem demonstrar os diferentes tópicos a serem explorados por meio do caso. Não se deve esquecer de que, em consonância com Howard e Stout (2006), notas de ensino construídas de modo 
descuidado constituem, ao lado da falta de informações robustas para dar suporte ao caso, a razão mais frequente para a rejeição de casos de ensino submetidos a periódicos e a eventos.

Finalmente, as notas de ensino também podem trazer indicações sobre o público para o qual o caso é destinado. Alguns autores apontam obstáculos para que se use o método do caso com estudantes de graduação, e aconselham que o método seja usado principalmente em pós-graduação. Weber e Kirk (2000) defendem que o método do caso pode ser usado com alunos de graduação; porém, nesses casos, é necessário realizar uma aula introdutória para explicar os objetivos e a dinâmica das aulas a partir do método.

\section{Elementos Utilizados para Análise dos Casos e Respectivas Notas de Ensino}

Com base na revisão de literatura, foram selecionados os elementos mais importantes a serem utilizados na análise dos casos e das notas de ensino. A tabela abaixo apresenta esses elementos com o objetivo de melhorar o entendimento das análises.

Tabela 1

\section{Elementos para a Análise dos Casos}

\begin{tabular}{|c|c|c|}
\hline Elementos & Aspectos observados & Referências \\
\hline Título & $\begin{array}{l}\text { Títulos neutros contendo o nome da } \\
\text { empresa. }\end{array}$ & $\begin{array}{l}\text { Leenders et al. (2001); Roesch } \\
\text { (2007). }\end{array}$ \\
\hline Protagonista do caso & $\begin{array}{l}\text { Perfil do protagonista que promova } \\
\text { aproximação com o leitor. }\end{array}$ & $\begin{array}{l}\text { Corey (1998); Franke (2011); } \\
\text { Leenders et al. (2001); Sebenius, } \\
2011 .\end{array}$ \\
\hline Tamanho do caso & $\begin{array}{l}\text { Os casos não devem ser muito } \\
\text { grandes. }\end{array}$ & $\begin{array}{l}\text { Abell (1997); Corey (1998); Ikeda } \\
\text { et al. (2005); Roberts (1999). }\end{array}$ \\
\hline Redação & $\begin{array}{l}\text { Redação imparcial, atraente e } \\
\text { utilizando o tempo verbal no } \\
\text { passado. }\end{array}$ & $\begin{array}{l}\text { Gil (2004); Leenders et al. (2001); } \\
\text { Roberts (1999); Roesch (2007); } \\
\text { Roesch e Fernandes ( 2007); } \\
\text { Sebenius (2011); Webb et al. (2005). }\end{array}$ \\
\hline Confidencialidade & $\begin{array}{l}\text { Quando necessário, mudança do } \\
\text { nome da empresa e/ou dos } \\
\text { personagens para manter a } \\
\text { confidencialidade. }\end{array}$ & $\begin{array}{l}\text { Roberts (1999); Roesch e Fernandes } \\
\text { (2007). }\end{array}$ \\
\hline Fontes de informação & $\begin{array}{l}\text { Indicação das fontes utilizadas para } \\
\text { a elaboração dos casos. / Utilização } \\
\text { de fontes múltiplas. }\end{array}$ & $\begin{array}{l}\text { Cappel e Schwager (2002); Corey } \\
\text { (1999); Gil, (2004); Ikeda et al. } \\
\text { (2005); Roberts (1999). }\end{array}$ \\
\hline Questões para discussão & $\begin{array}{l}\text { Notas de ensino devem trazer } \\
\text { questões para discussão. }\end{array}$ & $\begin{array}{l}\text { Lundberg e Winn (2005); Roberts } \\
\text { (1999). }\end{array}$ \\
\hline $\begin{array}{l}\text { Disciplinas nas quais o caso pode } \\
\text { ser programado }\end{array}$ & $\begin{array}{l}\text { Notas de ensino devem trazer } \\
\text { indicação de em quais disciplinas o } \\
\text { caso pode ser utilizado. }\end{array}$ & $\begin{array}{l}\text { Lundberg e Winn (2005) Roesch } \\
\text { (2007); Roesch e Fernandes (2007). }\end{array}$ \\
\hline Público-alvo & $\begin{array}{l}\text { Notas de ensino devem trazer } \\
\text { indicação de para qual público } \\
\text { destina-se o caso (ex. alunos de pós- } \\
\text { graduação). }\end{array}$ & $\begin{array}{l}\text { Roberts (1999); Roesch e Fernandes } \\
\text { (2007). }\end{array}$ \\
\hline
\end{tabular}


Tabela 1 (continuação)

\begin{tabular}{|c|c|c|}
\hline Elementos & Aspectos observados & Referências \\
\hline Indicação de bibliografia & $\begin{array}{l}\text { Notas de ensino devem trazer } \\
\text { indicação de bibliografia útil para os } \\
\text { professores que desejam usar o caso. }\end{array}$ & $\begin{array}{l}\text { Roberts (1999); Roesch e Fernandes } \\
\text { (2007). }\end{array}$ \\
\hline Material de apoio & $\begin{array}{l}\text { Indicação de outros materiais que } \\
\text { podem ser utilizados junto com o } \\
\text { caso. }\end{array}$ & $\begin{array}{l}\text { Cappel e Schwager (2002); Roberts } \\
\text { (1999). }\end{array}$ \\
\hline Parágrafo inicial & $\begin{array}{l}\text { O primeiro parágrafo deve } \\
\text { apresentar: o protagonista, as } \\
\text { principais questões, a empresa e } \\
\text { quando se dão os acontecimentos. }\end{array}$ & $\begin{array}{l}\text { Leenders et al. (2001); Roberts } \\
\text { (1999); Roesch (2007); Roesch e } \\
\text { Fernandes (2007); Swiercz e Ross } \\
\text { (2003). }\end{array}$ \\
\hline
\end{tabular}

\section{Metodologia}

Confiando na análise bibliométrica, o presente trabalho fundamentou-se em pesquisa exploratória de âmbito quantitativo descritivo (Vergara, 2010). A bibliometria propõe o uso de métodos quantitativos para investigar a produção escrita como elemento da comunicação do conhecimento (Costas, Leeuwen, \& Bordons, 2010; Mattos, 2004; Moretti \& Campanario, 2009). Assim, com o suporte de processos variados de levantamento, tratamento e apresentação de dados, busca-se compreender a evolução da produtividade em um campo de estudos (Araújo \& Alvarenga, 2011; Guedes \& Borschiver, 2005; Wallace, Fleet, \& Downs, 2011).

Em análises de base bibliométrica, os conjuntos de publicações definidos como alvos da pesquisa são estatisticamente mapeados por meio do monitoramento e da descrição dos registros a partir de suas frequências (Vanti, 2002). Para tanto, foram seguidas as diretrizes empírico-analíticas sugeridas por Lordsleem, Araújo, Oliveira e Alexandre (2009) para a investigação bibliométrica no campo da administração: descrever as características das publicações e dos autores mais produtivos, incluindo as instituições que se sobressaem na produção de casos de ensino. Todos os casos publicados no período selecionado foram integralmente apreciados, e as informações acerca de seus conteúdos - tanto no que diz respeito aos casos quanto às notas de ensino - foram incluídas na discussão. Assim, a análise bibliométrica empreendida no estudo transcende a contagem dos indicadores usualmente considerados em trabalhos semelhantes.

O período analisado na presente pesquisa compreendeu os anos de 2007 a 2011 . Optou-se por esse recorte uma vez que 2007 foi o primeiro ano em que os anais do Encontro da ANPAD incluíram casos de ensino; cabe destacar, entretanto, que algumas revistas acadêmicas já publicavam casos de ensino antes de 2007. Os eventos considerados para a pesquisa comportaram cinco edições do Encontro da ANPAD (EnANPAD) e três edições do Encontro de Ensino e Pesquisa em Administração e Contabilidade (EnEPQ). No que diz respeito aos periódicos, empreendeu-se uma busca por casos de ensino nas edições entre 2007 e 2011 das revistas de administração com maior expressividade acadêmica, ou seja, aquelas com conceito até B2 segundo a classificação da CAPES. Cabe destacar que só foram encontrados casos de ensino em duas publicações: a Revista Eletrônica de Administração (REAd) e a Revista de Administração Contemporânea (RAC). Segundo informações constantes nos sítios eletrônicos das próprias revistas, oportunamente confirmadas com os editores das mesmas por correio eletrônico, a maioria dos periódicos brasileiros não aceita submissões de casos para ensino.

Para a estruturação dos dados da pesquisa, foi elaborado um banco de dados em planilhas do MS Excel ${ }^{\circledR}$, tendo por base o ano de publicação, os autores, suas afiliações institucionais e o evento de veiculação dos casos, seguindo as recomendações de Leite (2008). Além dessas informações, foram 
incluídos nas planilhas os dados sobre a empresa retratada, o protagonista da narrativa, o tamanho do caso, a redação e as notas de ensino.

De acordo com Guedes e Borschiver (2005), em estudos de base bibliométrica, deve-se verificar quais são os autores e centros de pesquisa mais produtivos. Nesse sentido, buscou-se seguir a diretriz de Alvarado (2002) a respeito da contagem completa de autores, e creditar com uma contribuição cada autor principal e/ou secundário. Para obter informações sobre as instituições com as quais os autores mantêm vínculo, recorreu-se aos anais dos respectivos congressos e às edições das revistas em que os casos foram publicados, atribuindo-se o caso à instituição na qual o autor se encontrava no momento da publicação. Com relação aos autores que apareciam nos anais dos eventos como vinculados a mais de uma instituição, foi considerada para a análise a primeira instituição indicada.

Alguns casos publicados em anais de congressos posteriormente foram selecionados para integrar edições de revistas acadêmicas. Para que tais casos não fossem duplamente contados na análise, foi considerada somente a versão veiculada no periódico, pois, em geral, a versão da revista de um estudo acadêmico costuma agregar melhorias sugeridas por pares.

Como delimitação, o presente trabalho ateve-se aos casos de ensino publicados no período especificado. Cabe notar que não foram consideradas na análise pesquisas que tivessem casos de ensino por tema. Ao todo foram encontrados 122 casos de ensino, ressalvando-se que o único caso publicado na REAd já havia sido publicado em anais de congresso, bem como quatorze dos dezenove casos da RAC. Portanto, seguindo a lógica de não contar duplamente um caso que tivesse sido publicado em dois veículos, foi considerado um número total de 107 casos para a análise depois da remoção dos quinze que apareciam duas vezes na contagem. A Tabela 2 enumera os casos publicados em anais de congressos e em cada revista.

Tabela 2

Casos de Ensino Publicados em Periódicos e Anais de Congressos

\begin{tabular}{lc}
\hline Local da publicação & Número de casos de ensino publicados \\
\hline Anais do EnANPAD 2007 & 24 \\
Anais do EnANPAD 2008 & 18 \\
Anais do EnANPAD 2009 & 11 \\
Anais do EnANPAD 2010 & 17 \\
Anais do EnANPAD 2011 & 8 \\
Anais do EnEPQ 2007 & 0 \\
Anais do EnEPQ 2009 & 12 \\
Anais do EnEPQ 2011 & 12 \\
REAd & 1 \\
RAC & 19 \\
\hline
\end{tabular}

\section{Caracterização e Análise dos Casos}

No ano de 2007, a Anpad aceitou pela primeira vez casos de ensino em seu congresso principal, o EnANPAD. Naquela ocasião, os casos representaram 2,5\% do total de trabalhos aceitos para apresentação e posterior publicação nos anais do evento. No EnANPAD de 2008, esse volume caiu para $1,8 \%$ do total. Em 2009, a queda foi mantida e a representatividade dos casos de ensino no evento foi de $1,5 \%$. Registrou-se, entretanto, um aumento desse volume em 2010, quando os casos de ensino 
representaram 2\% do total de artigos no EnANPAD e, em 2011, uma nova queda foi registrada, e os casos representaram somente $1 \%$ do total. Já no que tange ao EnEPQ, na edição de 2007, nenhum caso de ensino foi publicado, mas, em 2009, constaram nos anais do encontro 12 casos, correspondentes a 14,5\% dos trabalhos totais. Na edição de 2011 do EnPQ, os casos representaram 12\% do total de artigos aprovados.

Em revistas acadêmicas brasileiras, a publicação de casos de ensino ainda é muito escassa. Somente dois dentre os periódicos mais conceituados da área publicaram casos de ensino no período avaliado, sendo que a REAd publicou apenas um caso, enquanto a RAC publicou 19 casos de ensino. Curiosamente, como Kruel e Santos (2008) já haviam apontado, alguns dos textos publicados como casos de ensino contêm, em suas conclusões, questionamentos e reflexões típicas de estudos de caso. Em alguns trabalhos, fica claro que os autores não são capazes de diferenciar casos de ensino de estudos de caso. A confusão fica mais patente ao se verificar que, em nove casos, aparece a expressão estudos de caso de ensino.

No que diz respeito aos autores dos casos publicados, cabe destacar que, ao todo, 231 acadêmicos assinaram os 107 casos analisados em autoria ou coautoria, porém somente 35 têm mais de um caso publicado, mostrando que a maioria das pessoas que publicam um caso não volta a escrever. Tal cenário parece refletir um aspecto levantado pela literatura: os autores escrevem casos quando têm a oportunidade de conseguir acesso fácil aos dados de alguma empresa, quando o ideal seria que a motivação para a elaboração de um caso viesse de necessidades pedagógicas (Roberts, 1999). A Tabela 3 mostra os autores brasileiros que mais publicaram casos no período analisado. Nessa tabela, foram listados todos os autores que publicaram três casos ou mais. Outros quinze autores contribuíram com dois casos cada.

Tabela 3

\section{Autores Mais Produtivos de Casos de Ensino}

\begin{tabular}{llc}
\hline Autores & Instituição & Número de casos \\
\hline Kleber Fossati Figueiredo & Instituto COPPEAD & 8 \\
Angela da Rocha & Instituto COPPEAD/PUC-Rio & 7 \\
Pelayo Munhoz Olea & Universidade de Caxias do Sul & 7 \\
Victor Manoel Cunha de Almeida & Instituto COPPEAD & 7 \\
Renato Cotta & Instituto COPPEAD & 6 \\
Ilan Avrichir & ESPM - São Paulo & 4 \\
Joyce Gonçalves Altaf & Universidade Estácio de Sá & 4 \\
Márcio Moutinho Abdalla & Universidade Estácio de Sá & 4 \\
Anne-Marie Maculan & COPPE-UFRJ & 3 \\
Lilian Aparecida Pasquini Miguel & PUC- Minas Gerais & 3 \\
Sylvia Maria Roesch & Universidade de Caxias do Sul & 3 \\
Maribel Carvalho Suarez & Instituto COPPEAD & 3 \\
Leticia Moreira Cosotti & Instituto COPPEAD & 3 \\
\hline
\end{tabular}

Guiada pelo propósito de traçar um panorama amplo da produção brasileira, esta pesquisa buscou elencar também as instituições de ensino superior (IES) que mais publicaram casos. Cabe reforçar que, para a atribuição dos casos às instituições, contabilizou-se a IES indicada por cada autor do caso no momento da publicação: um caso com três autores, por exemplo, contou para as três instituições, ou mais de uma vez para a mesma, se os autores estavam associados à mesma IES. Na Tabela 4, percebem-se duas intuições que se destacam como as mais produtivas: o Instituto 
COPPEAD, da Universidade Federal do Rio de Janeiro, e a Universidade de Caxias do Sul. Relacionando tal cenário com a revisão da literatura, é possível supor que, nessas IES, há apoio institucional para o uso do método do caso, o que pode ser um incentivo para que seus docentes tornem-se autores de casos muito produtivos.

Tabela 4

IES Mais Produtivas em Casos de Ensino

\begin{tabular}{lc}
\hline Instituição & Ocorrências \\
\hline COPPEAD & 56 \\
Universidade de Caxias do Sul & 38 \\
PUC-RIO & 28 \\
UNESA & 14 \\
UFRGS & 13 \\
IBMEC-Rio & 12 \\
Mackenzie & 9 \\
PUC-MINAS & 9 \\
\hline
\end{tabular}

Note-se que as seis instituições de ensino que mais produzem casos estão localizadas no Rio Grande do Sul ou no Rio de Janeiro, o que representa que esses dois estados são responsáveis pela grande maioria dos casos de ensino utilizados nas escolas de administração de todo o país. Devido à grande pluralidade brasileira, é importante que autores de instituições de outros estados também se dediquem à produção de casos locais e nacionais.

Ainda no começo das análises, foi possível perceber que a maioria dos títulos dos casos não segue as indicações da literatura, que preconiza a identificação dos casos somente pelo nome da empresa, para que sejam neutros (Roesch, 2007). Dos casos analisados, 18 têm o título composto apenas pelo nome da empresa, seguindo a recomendação da literatura, enquanto 27 títulos sequer contêm o nome da empresa e 8 títulos contêm elementos de humor o que não seria desejável (Leenders et al., 2001). Como exemplos, tem-se os seguintes títulos: Qual o tamanho do tubarão para o tanque de peixes? Um caso de ensino envolvendo conflitos em canais de distribuição (Costa, Mesquita, Fernandes, \& Caixeta, 2011); Caso WebBusiness: Vender ou não Vender, eis a Questão (Avrichir, Almeida, \& Andreassi, 2011); Oh que Droga: Não Acredito que Levei uma Multa! (Muzzio, 2008). Contudo, acredita-se que títulos engraçados possam aproximar o caso dos alunos.

Analisando os casos no que diz respeito às áreas do conhecimento administrativo para as quais eles possam ser aproveitados, percebe-se que a maioria dos casos é apontada por seus autores como úteis primordialmente para a área de estratégia (45 casos) e depois para marketing ( 35 casos). Por outro lado, existem áreas desprivilegiadas que não contam com nenhum caso, e outras que contam com poucos casos, tais como gestão de pessoas, logística e finanças. Buscou-se fazer a relação entre as áreas mais apontadas e os autores mais produtivos. Foi possível perceber que esses autores, na maioria das vezes, escrevem casos para serem utilizados nas mesmas disciplinas e que o grande número de casos nas áreas de estratégia e de marketing não pode ser atribuído aos autores que mais escrevem casos e sim aos que escreveram somente um caso no período analisado. A Tabela 5, apresentada a seguir, mostra quantos casos se propõem a serem usados em cada área. Cabe observar que a soma dos números apresentados na tabela é maior do que o número de casos analisados, uma vez que um mesmo caso pode ser indicado pelos autores como sendo útil para mais de uma área.

Note-se que, em quatorze dos casos analisados, os autores não indicam em quais disciplinas os casos podem ser usados, o que dificulta sua escolha por professores que desejem utilizá-los em suas 
aulas. A literatura aponta que é fundamental indicar, nas notas de ensino, as matérias em que o caso poderá ser usado, assim como seus objetivos educacionais (Roesch, 2007).

Tabela 5

Distribuição dos Casos por Áreas Temáticas

\begin{tabular}{lc}
\hline Área & Casos \\
\hline Estratégia & 45 \\
Marketing & 35 \\
Empreendedorismo & 14 \\
Administração internacional & 8 \\
Logística e Produção & 6 \\
Gestão de Pessoas & 6 \\
Finanças & 5 \\
Ética & 5 \\
Administração Pública & 2 \\
\hline
\end{tabular}

A literatura apregoa que há muitas dificuldades para utilizar o método do caso em cursos de graduação (Weber \& Kirk, 2000). Não obstante, 43 dos casos analisados dizem ser adequados para o aproveitamento em turmas de graduação, sem tecer consideração alguma a respeito de possíveis dificuldades a serem enfrentadas para essa utilização. Grande parte desses casos indicados para serem usados em graduação apresenta problemas nas notas de ensino. Em alguns não há indicação de questões para discussão e, em outros, não fica claro como foram obtidas as informação para a elaboração dos casos.

Nas notas de ensino de 21 casos, não foi especificado o público para o qual eles eram direcionados. Outro problema percebido nas notas de ensino dos casos analisados foi a falta de indicação de quais foram as fontes utilizadas para a elaboração dos casos. Em 37 casos, não foi registrada essa informação, que é fundamental para que se entenda de onde foram tirados os dados, se eles retrataram apenas a visão de um executivo da empresa ou se o autor teve acesso a documentos, por exemplo. Roesh (2007) assevera que a indicação das fontes é fundamental para que o caso tenha credibilidade. Ainda no que diz respeito às fontes nas quais o autor se baseou para escrever o caso, nota-se que a fonte mais comumente utilizada foi a entrevista, presente em 62 dos 107 casos. Documentos da empresa só foram analisados pelos autores de 17 casos, e, em doze casos, foram utilizadas apenas fontes secundárias. Esse cenário não reflete o que é preconizado pela literatura, que defende o suporte de múltiplas fontes para que o caso não traga apenas o ponto de vista da empresa (Corey, 1999; Ikeda, Veludo-de-Oliveira, \& Campomar, 2005; Roberts, 1999; Sebenius, 2011). Altaf, Troccoli, Abdalla, Ribas e Corrêa (2009, p. 7), por exemplo, afirma que utilizaram múltiplas fontes de obtenção de dados:

O caso foi elaborado a partir de entrevistas em profundidade com os três fundadores da empresa. Além disso, documentos institucionais, relatórios sobre o setor e reportagens sobre o segmento foram utilizados na complementação das informações necessárias para a redação do caso.

Ainda no que tange ao conteúdo das notas de ensino, um elemento importante para que as notas cumpram seu papel de auxiliar o professor é a indicação de perguntas que possam servir como guias para os debates. Embora a maior parte das notas analisadas trouxesse perguntas, em treze delas não havia esse ponto fundamental. 
A despeito de existir na literatura a indicação de que os casos de ensino sejam utilizados conjuntamente com outras mídias, tais como notícias de jornal e filmes (Roberts, 1999), nas notas de ensino de somente três casos havia indicações de elementos complementares a serem utilizados na dinâmica com os casos.

A literatura também havia apontado que vários autores de casos são obrigados a mudar o nome da empresa, para que seja mantida a confidencialidade exigida por muitos executivos que aceitam fornecer informações para a elaboração do caso (Roesch \& Fernandes, 2007). A análise dos casos revelou essa camuflagem em muitos textos, porém, somente em dez deles a mudança do nome da empresa foi explicitada nas notas de ensino.

A Tabela 5 traz um resumo de alguns elementos os quais não estão presentes em todos os casos analisados e que são muito importantes para as notas de ensino cumprirem com o objetivo de auxiliar o professor que optar por aproveitar os casos em suas atividades letivas. Cabe observar que muitos casos apresentam mais de um desses problemas em suas notas de ensino. Também foi possível notar que os casos mais recentes apresentam menos esses problemas do que os escritos há muito tempo, e que os casos publicados em revistas tendem a ter notas de ensinos mais completas do que os presentes em anais de congressos.

Tabela 6

\section{Problemas Recorrentes nas Notas de Ensino}

\begin{tabular}{lc}
\hline Não apresentam ... & \% do total dos casos \\
\hline ... indicações de elementos complementares a serem utilizados com os casos. & $97 \%$ \\
$\ldots$ elementos sobre possíveis mudanças em informações para manter a confidencialidade. & $91 \%$ \\
$\ldots$ fontes dos dados utilizadas para a elaboração do caso. & $35 \%$ \\
$\ldots$ sugestões sobre o público para o qual o caso deve ser utilizado. & $20 \%$ \\
$\ldots$ orientações acerca das disciplinas nas quais o caso pode ser aproveitado. & $13 \%$ \\
$\ldots$ perguntas para servir como guias nos debates. & $12 \%$ \\
$\ldots$ indicações de referências bibliográficas. & $10 \%$ \\
\hline
\end{tabular}

Analisando o modo como foram redigidos os casos, foi possível perceber que a maior parte deles apresenta uma história como suporte narrativo. O fato de o autor contextualizar os dados apresentados e realizar apresentações dos personagens presentes nos casos faz com que a narrativa se torne atraente para o aluno. A literatura enfatiza a importância de o leitor ser envolvido pela história do caso (Brennan \& Ahmad, 2005; Ellet, 2007; Greenhalgh, 2007; Roesch \& Fernandes, 2007). Cabe ressaltar, porém, que 23 dos casos analisados apresentam somente os dados, sem nenhuma história como suporte narrativo.

Outra forma de aproximar o caso dos alunos é escolher um protagonista com o qual eles possam se identificar (Leenders et al., 2001). Por esse motivo, fez-se um levantamento do perfil dos protagonistas apresentados nos casos. A característica mais notória foi que somente em 11 deles o protagonista é do sexo feminino A Tabela 6 mostra o cargo ou a posição hierárquica que os protagonistas dos casos ocupam nas empresas retratadas. A maior parte dos protagonistas são os próprios donos das empresas, seguidos por sócios e diretores. A utilização exclusiva de casos nos quais o decisor é um alto executivo pode passar ao aluno a falsa ilusão de que outros atores organizacionais não são importantes para a tomada de decisão. Ressalte-se ainda que, em 31 casos, não houve nenhuma indicação do cargo ocupado pelo protagonista. Diante desse cenário, acredita-se que novos casos poderiam incluir protagonistas femininas e outras perspectivas de decisão dentro da empresa. Essa diversificação poderia ampliar as situações e as possibilidades de discussão para aqueles que utilizam casos de ensino. 
Para Roberts (1999) e Roesch (2007), a apresentação do protagonista deve ocorrer no primeiro parágrafo, mas apenas 20 dentre os casos analisados seguiram tal diretriz. A literatura aponta ainda que, além da caracterização desse personagem, o primeiro parágrafo deve apresentar a empresa e a situação-problema (Swiercz \& Ross, 2003), e assim foi em 62 casos. Em sete dos casos analisados, a empresa não foi apresentada no primeiro parágrafo.

Tabela 7

\section{Cargos ou Posições Ocupados pelos Protagonistas dos Casos}

\begin{tabular}{lc}
\hline Protagonista & Casos \\
\hline Dono & 27 \\
Sócio & 13 \\
Diretor & 11 \\
Presidente & 10 \\
Gerente & 8 \\
CEO & 3 \\
Assessor & 1 \\
Fundador & 1 \\
Médico & 1 \\
Vice-presidente & 1 \\
Sem identificação & 31 \\
\hline
\end{tabular}

Além da preocupação com o conteúdo do caso, a literatura indica que o autor deve atentar para o tamanho do texto, haja vista que casos muito grandes podem causar desinteresse por parte dos alunos (Ikeda et al., 2005). Abell (1997), por instância, defende que os casos devem ter no máximo de oito a dez páginas. A Figura 1 apresenta o número de páginas dos casos analisados na presente pesquisa, sem contar as notas de ensino. Percebe-se que a maior parte tem nove páginas, o que está de acordo com a literatura. Entretanto, 22 casos têm mais do que 10 páginas, o que pode configurar um problema, tanto por exigir muito do aluno, quanto porque pode significar que as notas de ensino não cobrem tudo o que deveriam, diante do limite máximo de páginas imposto pela publicação ou pelo congresso.

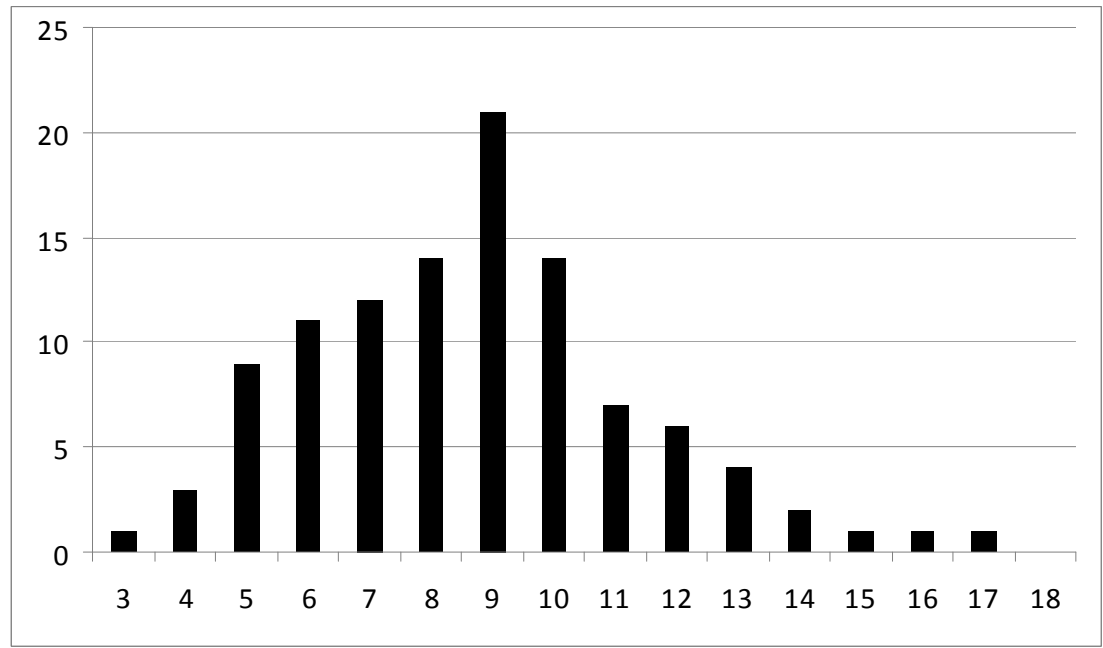

Figura 1. Número de Páginas dos Casos Analisados. 


\section{Diretrizes Gerais para Autores de Casos de Ensino no Brasil}

Com base na revisão de literatura apresentada previamente e na análise da produção brasileira de casos de ensino entre os anos de 2007 e 2011, foram elencadas 16 diretrizes com o propósito de fornecer orientações gerais para acadêmicos brasileiros que desejem produzir casos de ensino em administração. Tais diretrizes e as principais fontes utilizadas para elaborá-las estão relacionadas na Tabela 8.

Tabela 8

\section{Diretrizes Gerais para Acadêmicos do Campo de Administração Interessados em Produzir de Casos de Ensino no Brasil.}

1. Submeter os casos aceitos para apresentação em Achados da pesquisa. congressos aos processos seletivos para publicação nos periódicos brasileiros que aceitam casos de ensino.

2. Produzir casos que possam ser utilizados em disciplinas das áreas de gestão de pessoas, finanças e logística/operações, uma vez que a produção de casos brasileiros que atendam a essas áreas é muito escassa.

3. Produzir casos que levantem questões relacionadas à dimensão da ética e da responsabilidade social corporativa, de modo que possam colocar o aluno em contato com importantes debates éticos em diversas situações empresariais.

4. Indicar sempre para quais disciplinas e para que nível (graduação ou pós-graduação) o caso é adequado, de maneira a descomplicar a escolha dos casos pelos professores.

5. Utilizar fontes de informação múltiplas para a elaboração dos casos, para que estes sejam mais completos e reúnam vários pontos de vista.

6. Sempre explicitar que fontes de informações foram usadas na pesquisa para elaborar o caso, de modo que o texto seja mais crível e para orientar o professor que utilizará o caso.

7. Incluir perguntas que possam servir como guia para o professor no momento que ele estiver utilizando o caso em sala de aula.

8. Apontar materiais extras, tais como filmes, reportagens de jornal e sites da internet que possam ser utilizados para complementar o trabalho com o caso.

9. Deixar claro nas notas de ensino se o nome da empresa é fictício; tal informação não precisa ser transmitida aos alunos, mas pode ser importante para os professores na preparação da aula.

10. Manter a preocupação de escrever o caso de forma envolvente, com uma narrativa que agrade aos alunos, para aumentar as chances de que eles participem das discussões.

Achados da pesquisa.

Achados da pesquisa.

Roberts (1999); Roesch e Fernandes (2007); achados da pesquisa.

Corey (1999); Gil, (2004); Ikeda et al. (2005); Roberts (1999); achados da pesquisa.

Corey (1999); Gil, (2004); Ikeda et al. (2005); Roberts (1999); achados da pesquisa.

Roberts (1999); achados da pesquisa.

Roberts (1999); achados da pesquisa.

Roesch \& Fernandes (2007); achados da pesquisa.

Gil (2004); Leenders et al. (2001); Roberts (1999); Roesch (2007); Roesch e Fernandes ( 2007); Sebenius (2011); Webb et al. (2005); achados da pesquisa. 


\section{Tabela 8 (continuação)}

11. Escrever os casos no passado para que o caso seja Gil (2004); Leenders et al. (2001); Roberts (1999); menos datado, podendo assim ser utilizado por mais tempo.

12. Adequar o perfil do protagonista da narrativa ao perfil dos alunos para os quais o caso é destinado, de modo a favorecer a identificação e a empatia dos discentes com o decisor do caso.

13. Preparar um primeiro parágrafo rico em forma e conteúdo, caracterizando o personagem, a empresa e apresentando a situação problema.

14. Atentar para a diferenciação entre a produção de um caso de ensino e a elaboração de um estudo de caso; para que o texto seja um caso de ensino é fundamental, por exemplo, que se defina um objetivo educacional.

15. Sem detrimento do conteúdo, privilegiar casos mais curtos, principalmente se o objetivo for utilizar o caso com alunos de graduação, pois o tempo investido na leitura do caso geralmente é suprimido do tempo dedicado à sua análise.

16. Buscar apoio institucional para a adoção sistemática do método do caso em classe, uma vez que tal suporte parece ser fundamental para que os professores disponham da estrutura necessária para escrever casos e para utilizar o método de forma efetiva.
Roesch (2007); Roesch e Fernandes ( 2007); Sebenius

(2011); Webb et al. (2005); achadas da pesquisa.

Franke, (2011); Sebenius, (2011); achados da pesquisa.

Leenders et al. (2001); Roberts (1999); Roesch (2007); Roesch e Fernandes (2007); Swiercz e Ross (2003); achados da pesquisa.

Kruel e Santos (2008); achados da pesquisa.

Abell (1997); Corey (1998); Ikeda et al. (2005);

Roberts (1999); achados da pesquisa.

Achados da pesquisa.

\section{Considerações Finais}

O presente artigo teve como objetivo, a partir de um panorama da produção dos casos brasileiros publicados entre 2007 e 2011, propor diretrizes para acadêmicos brasileiros em administração que desejem produzir casos de ensino. Cabe destacar que a elaboração do artigo teve como principal motivação contribuir para aumentar a produção de casos brasileiros adequados ao método de ensino ativo. Estabelecido a partir de uma análise de base bibliométrica, esse panorama apontou, inicialmente, que ainda são poucas as revistas acadêmicas que aceitam publicar casos de ensino e que, mesmo aquelas que os aceitam, não costumam publicar casos com frequência, à exceção da RAC. A maior aceitação de casos por periódicos parece configurar um caminho para estimular uma maior produção de casos de ensinos brasileiros.

É importante que o desenvolvimento de casos de ensino com foco em empresas brasileiras ganhe envergadura, para que os docentes brasileiros tenham à sua disposição casos que retratem a realidade de seus alunos, considerando que uma das principais críticas dirigidas ao método do caso é precisamente a de que o cenário retratado nos casos não corresponde à realidade brasileira. Destaca-se que, dentre os casos analisados no presente estudo, foram encontradas empresas estrangeiras e até mesmo casos escritos em inglês, o que não parece atender as demandas por casos que retratem a realidade das empresas nacionais. A maior parte dos casos analisados apresenta problemas nas notas de ensino, as quais se mostraram incompletas na maioria das vezes. Sendo assim, uma indicação geral para os autores de casos é de que aumentem a atenção voltada para a qualidade das notas de ensino. Muitas vezes os autores se preocupam em produzir casos muito extensos e se esquecem da importância das notas de ensino, que são fundamentais para que outros professores possam usar o caso 
em suas aulas (Corey, 1999; Gil, 2004; Roesch, 2007). Os achados da pesquisa apontam para a necessidade de maior atenção por parte dos autores na elaboração das notas de ensino. Esse alerta parece ser fundamental para que os casos produzidos por acadêmicos brasileiros sejam considerados como de boa qualidade e possam ser utilizados.

Para que seja possível atingir os objetivos didático-pedagógicos estipulados pela adoção do método do caso, torna-se crucial que os alunos participem das discussões. A partir da presente análise, foi possível perceber que algumas características dos casos podem ser ineficazes em prender a atenção dos alunos, fazendo com que a participação nos debates seja insuficiente para o aprendizado. Dentre essas características, destacam-se: o predomínio de protagonistas do sexo masculino ocupando cargos hierárquicos muito elevados, o que leva os alunos a não se identificarem com o personagem central do caso; narrativas desinteressantes somente com fatos e sem histórias envolventes, além de textos exageradamente longos. Sendo assim, os achados da pesquisa apontam para a necessidade de mudanças na produção brasileira de casos para que esses tenham maior atratividade, tanto do ponto de vista dos professores quanto dos alunos.

Roesch e Fernandes (2007) defendem que os casos deveriam ser testados para que os alunos que participassem do teste pudessem apontar ambiguidades, excesso de dados ou falta de informação, de maneira que os autores modificassem a narrativa no que fosse preciso. Essa posição leva ao questionamento de que talvez fosse importante que os casos fossem testados antes mesmo de serem veiculados ou publicados. Esse teste - cujos resultados poderiam ser indicados nos notas de ensino configuraria um passo importante para que a versão publicada do caso fosse adequada aos objetivos pedagógicos estabelecidos.

Como sugestão para pesquisas futuras, propõe-se que sejam produzidos estudos que busquem analisar as experiências de professores brasileiros com o uso dos casos nacionais publicados. Por meio de tais pesquisas, e de sua comparação com aquelas já existentes acerca do uso de casos estrangeiros, seria possível apontar novos caminhos para a produção nacional e para a aplicação dos casos em instituições brasileiras de ensino superior.

\section{Referências}

Abell, D. (1997). What makes a good case? ECCHO - The Newsletter of the European Case Clearing House, 17(1), 4-7.

Almeida, V., \& Figueiredo, K. (2007, novembro). Evidências empíricas da importância da preparação individual no processo de aprendizagem pelo método do caso: um experimento de campo com alunos de pós-graduação. Anais do Encontro de Ensino e Pesquisa em Administração $e$ Contabilidade, Recife, PE, Brasil, 1.

Altaf, J., Troccoli, I., Abdalla, M., Ribas, J., \& Corrêa, B. (2009, novembro). Grupo rei da sucata as dores do crescimento: da sucata ao ouro? Anais do Encontro de Ensino e Pesquisa em Administração e Contabilidade, Curitiba, PR, Brasil, 2.

Alvarado, R. (2002). A lei de Lotka na bibliometria brasileira. Ciência da Informação, 31(2), 14-20. doi: $10.1590 /$ S0100-19652002000200002

Ambrosini, V., Bowman, C., \& Collier, N. (2010). Using teaching case studies for management research. Strategic Organization, 8(3), 206-229. doi: 10.1177/1476127010374254

Apaydin, M. (2008). Making a case for the case method in Turkey. Journal of Management Development, 27(7), 678-692. doi: 10.1108/02621710810883599 
Araújo, R., \& Alvarenga, L. (2011). A bibliometria na pesquisa científica da pós-graduação brasileira de 1987 a 2007. Revista Eletrônica de Biblioteconomia e Ciência da Informação, 16(31), 51-70. doi: $10.5007 / 1518-2924$

Austin, J. (1993). Teaching notes: communicating the teacher's wisdom [Folheto $\mathrm{N}^{\circ}$ 5]. Harvard Business School Publish Division, Boston, MA,USA.

Avrichir, I., Almeida, V., \& Andreassi, T. (2011). Caso WebBusiness: vender ou não vender, eis a questão. Revista de Administração Contemporânea, 15(4), 776-789. Recuperado de http://www.scielo.br/pdf/rac/v15n4/a14v15n4.pdf. doi: 10.1590/S1415-65552011000400014

Banning, K. (2003). The effect of the case method on tolerance for ambiguity. Journal of Management Education, 27(5), 556-567. doi: 10.1177/1052562903252652

Bess, J. (2004). Using cases in higher education: a guide for faculty and administrators. The Review of Higher Education, 27(2), 262-264. doi: 10.1353/rhe.2003.0050

Böcker, F. (1987). Is case teaching more effective than lecture teaching in business administration? An exploratory analysis. Interfaces, 17(5), 64-71. doi: 10.1287/inte.17.5.64

Bontis, N., Hardie, T., \& Serenko, A. (2009). Techniques for assessing skills and knowledge in a business strategy classroom. International Journal of Teaching and Case Studies, 2(2), 162-180. doi: 10.1504/IJTCS.2009.031060

Brennan, R., \& Ahmad, S. (2005). Using case studies in management education: the student perspective. International Journal of Management Education, 4(3), 21-30.

Cappel, J., \& Schwager, P. (2002). Writing IS teaching cases: guidelines for JISE Submission. Journal of of Information Systems Education, 13(4), 287-294.

Christensen, C. M. (1991). Every student teaches every teacher learns: the reciprocal gift of discussion teaching. In C. Christensen, D. Garvin, \& A. Sweet (Orgs.), Education for Judment (pp. 99119). Boston: Harvard Business School Press.

Christensen, C. M., \& Carlile, P. (2009). Course research: using the case method to build and teach management theory. The Academy of Management Learning and Education, 8(2), 240-251.

Corey, E. (1998). Case method teaching [Folheto No 9-581-058]. Harvard Business School Publishing Division, Boston, MA, USA.

Corey, E. (1999). Whiting cases and teaching notes [Folheto $\mathrm{N}^{\circ}$ 9-3999-077]. Harvard Business School Publishing Division, Boston, MA, USA.

Costa, C., Filho, Mesquita, J., Fernandes, R., \& Caixeta, R. (2011, novembro). Qual o tamanho do tubarão para o tanque de peixes?1 Um caso de ensino envolvendo conflitos em canais de distribuição. Anais do Encontro de Ensino e Pesquisa em Administração e Contabilidade da $A N P A D$, João Pessoa, PB, Brasil, 3.

Costas, R., Leeuwn, T., \& Bordons, M. (2010). A bibliometric classificatory approach for the study and assessment of research performance at the individual level: The effects of age on productivity and impact. Journal of the American Society for Information Science and Technology, 61(8), 1564-1581. doi: 10.1002/asi.21348

Duarte, F. (2010). Addressing student cynicism through transformative learning. Journal of University Teaching \& Learning Practice, 7(1), 1-15.

Ellet, W. (2007). The case study handbook: how to read, discuss and write persuasively about cases. Boston: Harvard Business School Press. 
Erskine, J., Leenders, M., \& Mauffette-Leenders, L. (2003). Teaching with cases (3th ed.). Ontario: Richard Ivey School of Business.

Ferlie, E., McGivern, G., \& Moraes, A. (2010). Developing a public interest school of management [Special Issue]. British Journal of Management, 21, s60-s70.

Franke, V. (2011). Decision-making under uncertainty: using case studies for teaching strategy in complex environments. Journal of Military and Strategic Studies, 13(2), 1-21.

Garvin, D. (1991). Barriers and gateways to learning. In C. Christensen, D. Garvin, \& A. Sweet (Orgs.), Education for Judment (pp. 3-13). Boston: Harvard Business School Press.

Garvin, D. (2007). Teaching executives and teaching MBAs: reflections on the case method. The Academy of Management Learning and Education, 6(3), 364-374.

Gil, A. (2004). Elaboração de casos para o ensino de administração. Contextus - Revista Contemporânea de Economia e Gestão, 12(2), 7-16.

Greenhalgh, A. (2007). Case method teaching as science and art: a metaphoric approach and curricular application. Journal of Management Education, 31(2), 181-194.

Guedes, V., \& Borschivers, S. (2005, outubro). Bibliometria: uma ferramenta estatística para a gestão da informação e do conhecimento, em sistemas de informação, de comunicação e de avaliação científica e tecnológica. Anais do Encontro Nacional de Ciências da Informação, Salvador, BA, Brasil, 6.

Hallowell, R., \& Hansen, A. (2000). Sothebys.com [Folheto No 9-800-387 2000]. Harvard Business Review, Boston, MA, USA.

Howard, T., \& Stout, D. (2006). Reasons accounting case/instructional resource papers are rejected for publication. Journal of Accounting Education, 24(1), 1-15. doi: 10.1016/j.jaccedu.2006.04.002

Iizuka, E. S. (2008, setembro). O método do caso de Harvard: Reflexões sobre sua pertinência ao contexto brasileiro. Anais do Encontro da Associação Nacional de Pós-Graduação e Pesquisa em Administração, Rio de Janeiro, RJ, Brasil, 32.

Ikeda, A., Veludo-de-Oliveira, T., \& Campomar, M. (2005). A tipologia do método do caso em administração: usos e aplicações. Organizações \& Sociedade, 12(34), 141-159.

Kopczak, L., \& Lee, H. (1996). Hewlett-Packard: Deskjet Printer Supply Chain. Stanford Teaching Cases.

Kruel, A., \& Santos, C. (2008, setembro). Estudo de caso ou caso para ensino? Uma análise de casos produzidos em um curso de especialização no Sul do Brasil. Anais do Encontro Nacional da Associação Nacional de Pós-Graduação e Pesquisa em Administração, Rio de Janeiro, RJ, Brasil, 32.

Leenders, M. R., Mauffette-Leenders, L. A., \& Erskine, J. A. (2001). Writing cases (4th ed.). Ontario: Richard Ivey School of Business.

Leite, G. (2008). Padrões de produtividade de autores em periódicos e congressos na área de contabilidade no Brasil: um estudo bibliométrico. Revista de Administração Contemporânea, 12(2), 533-554. doi: 10.1590/S1415-65552008000200011

Levin, B. (1995). Using the case method in teacher education: the role of discussion and experience in teacher's thinking about cases. Teaching \& Teacher Education, 11(1), 63-79. doi: 10.1016/0742-051X(94)00013-V 
Lordsleem, N., Araújo, R., Oliveira, B., \& Alexandre, M. (2009). Ensino e pesquisa em administração: um estudo bibliométrico de publicações do EnANPAD (2001-2008). Revista Ciências Administrativas, 15(2), 356-378.

Lundberg, C., \& Winn, J. (2005). The great case teaching notes debate. Journal of Management Education, 29(2), 268-283. doi: 10.1177/1052562904264284

Machado, A., \& Callado, A. (2007, novembro). Precauções na adoção do método de estudo de caso para o ensino de administração. Anais do Encontro de Ensino e Pesquisa em Administração e Contabilidade da ANPAD, Recife, PE, Brasil, 1.

Mahboubian, M. (2010). Educational aspects of business simulation softwares. Procedia - Social and Behavioral Sciences, 2(2), 5403-5407. doi: 10.1016/j.sbspro.2010.03.881

Mattos, P. (2004). "Bibliometria": a metodologia acadêmica convencional em questão. $R A E$ eletrônica, 3(2), 1-6. Recuperado de http://www.scielo.br/pdf/raeel/v3n2/v3n2a16.pdf. doi: $10.1590 /$ S1676-56482004000200016

Mauffette-Leenders, L., Erskine, J., \& Leenders, M. (2007). Learning whith cases (4th ed.). Ontario: Richard Ivey School of Business.

Moretti, S., \& Campanario, M. (2009). A produção intelectual brasileira em responsabilidade social empresarial - RSE sob a ótica da bibliometria [Edição Especial]. Revista de Administração Contemporânea, $13, \quad 68-89 . \quad$ Recuperado de http://www.scielo.br/pdf/rac/v13nspe/a06v13nspe.pdf. $\quad$ doi: 10.1590/S141565552009000500006

Mumford, A. (2005). The case method - does learning theory matter? Development and Learning in Organizations, 19(4), 17-19. doi: 10.1108/14777280510700344

Muzzio, H. (2008, setembro). Oh que droga: não acredito que levei uma multa! Anais do Encontro Nacional da Associação Nacional de Pós-Graduação e Pesquisa em Administração, Rio de Janeiro, RJ, Brasil, 32.

Oros, A. (2007). Let's debate: active learning encourages student participation and critical thinking. Journal of Political Science Education, 3(3), 293-311. doi: 10.1080/15512160701558273

Pinnington, A. (2005). Learning in a competitive field: MBA students' improvised case studies of IHRM. The International Journal of Human Resource Management, 16(4), 615-631. doi: $10.1080 / 09585190500051761$

Roberts, M. (1999). Developing teaching cases [Folheto N ${ }^{\circ}$ 9-900-001]. Harvard Business School Publishing, Boston, MA, USA.

Roesch, S. (2007). Notas sobre a construção de casos para ensino (Caso de Ensino em Administração). Revista de Administração Contemporânea, 11(2), 213-234. Recuperado de http://www.scielo.br/pdf/rac/v11n2/a12v11n2.pdf. doi: 10.1590/S1415-65552007000200012

Roesch, S., \& Fernandes, F. (2007). Como escrever casos para o ensino de administração. São Paulo: Atlas.

Rosier, G. (2002). Using reflective reports to improve the case method. Journal of Management Development, 21(8), 589-597. doi: 10.1108/02621710210437563

Sebenius, J. (2011). Developing superior negotiation case studies. Negotiation Journal, 27(1), 69-85. doi: 10.1108/02621710210437563 
Siciliano, J., \& McAleer, G. (1997). Increasing student participation in case discussion: using the MICA method in strategic management courses. Journal of Management, 21(2), 209-219. doi: $10.1177 / 105256299702100206$

Suarez, M., \& Casotti, L. (2004, setembro). Um novo professor para um novo aluno? Uma discussão sobre a utilização do método do caso. Anais do Encontro Nacional da Associação Nacional de Pós-Graduação e Pesquisa em Administração, Curitiba, PR, Brasil, 28.

Swiercz, P. M., \& Ross, K. T. (2003). Rational, human, political and symbolic text in Harvard Business School cases: a study of structure and content. Journal of Management Education, 27(4), 407-430. doi: 10.1177/1052562903252511

Vanti, N. A. P. (2002). Da bibliometria à webometria: uma exploração conceitual dos mecanismos utilizados para medir o registro da informação e a difusão do conhecimento. Ciência da Informação, 31(2), 152-162.

Vergara, S. (2003). Repensando a relação ensino-aprendizagem em Administração: argumentos teóricos, práticas e recursos. Organizações \& Sociedade, 10(28), 134-146.

Vergara, S. (2010). Projetos e relatórios de pesquisa em administração (12a ed.). São Paulo: Atlas.

Wallace, D., Fleet, C., \& Downs, L. (2011). The research core of the knowledge management literature. International Journal of Information Management, 31(1), 14-20. doi: 10.1016/j.ijinfomgt.2010.10.002

Wang, S., \& Wang, H. (2011). Teaching design thinking through case analysis: joint analyctical process. Decision Sciences Journal of Innovative Education, 9(1), 113-118. doi: 10.1111/j.15404609.2010.00295.x

Webb, H., Gill, G., \& Poe, G. (2005). Teaching with the case method online: pure versus hybrid approaches. Decision Sciences Journal of Innovative Education, 3(2), 223-250. doi: 10.1111/j.1540-4609.2005.00068.x

Weber, M., \& Kirk, D. (2000). Teaching teachers to teach cases: it's not what you know, it's what you ask. Marketing Education Review, 10(2), 59-67. 